\title{
Sexual transmission of gonorrhoea is facilitated by other bacteria
}

Infections with the bacterium Neisseria gonorrhoea are among the commonest of all sexually transmitted infections, and, in the past few years, antibiotic-resistant strains of this bacterium have started to emerge. Now researchers have demonstrated a key role of the female cervicovaginal microbiota in enhancing the infectivity, and thus the risk of female-to-male sexual transmission of this bacterium.

Lead author Michael A. Apicella explains: "In men, urethral-cell entry depends on the engagement of a terminal galactose on a gonococcal glycolipid called the lipooligosaccharide (LOS) by the asialoglycoprotein receptor. This entry process is dependent on the availability of a terminal galactose on the LOS." Previous studies in humans have shown that the LOS must contain a terminal galactose; however, during the infection process this terminal is normally capped with sialic acid. Apicella highlights "This led us to examine the question, do the cervical secretions contain sialidases, and if so, can they remove sialic acid from the gonococcal LOS?"

To answer this question, researchers investigated cervical and urethral secretions from a total of 57 women and seven men for the presence of sialidase activity. Analysis of these secretions revealed high levels of sialidase activity in samples taken from women who attended an STI clinic - 71\% of secretion samples from women who attended such clinics had levels of sialidase activity above the limits of detection by the assay used - compared with moderate sialidase activity in samples provided by 3 of 11 women that attended a gynaecology clinic.

The relevance of this finding was confirmed by the observation that this level of sialidase activity was able to remove the Neu5ac penultimate galactose moiety in samples from women with N. Gonorrhoea infections, and these findings were further confirmed using in vitro sialidase assays.

PCR analysis revealed the presence of DNA encoding microbial sialidase in

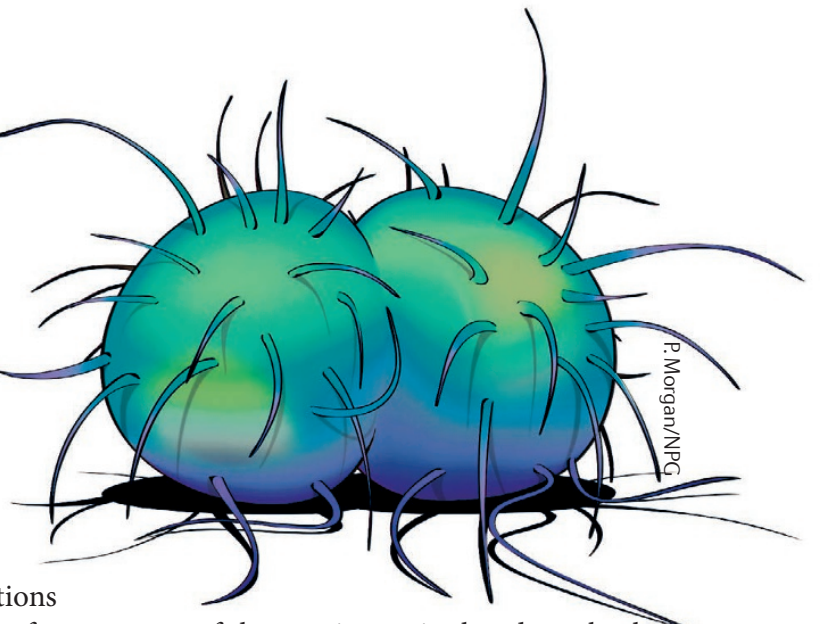

many of the cervicovaginal and urethral secretions tested, including from Gardnerella vaginalis, Prevotella bivia or Megasphaera spp, implying that sialidase activity arises from other members of the cervicovaginal microbiota.

This work highlights a role of the vaginal microbiota in the sexual transmission of $N$. Gonorrhoea infections. When asked about future directions of this work, Apicella speculates "The work raise the question as to whether other bacterial and/or host enzymes can alter the surface antigens of the gonococcus, and might make vaccine development challenging".

Peter Sidaway

ORIGINAL ARTICLE Ketterer, M. R.et al. Desialylation of Neisseria gonorrhoeae lipooligosaccharide by cervicovaginal microbiome sialidases: the potential for enhancing infectivity in men.J. Infect. dis. http://dx.doi.org/10.1093/infdis/jiw329 (2016) 